Research Paper

\title{
The gamma-glutamyl transpeptidase to platelet ratio for non- invasive assessment of liver fibrosis in patients with chronic hepatitis B and non-alcoholic fatty liver disease
}

\author{
Qiang Li ${ }^{1,2}$, Chuan Lu ${ }^{1}$, Weixia Li ${ }^{1}$, Yuxian Huang ${ }^{1,2}$, Liang Chen ${ }^{1}$ \\ ${ }^{1}$ Department of Hepatitis, Shanghai Public Health Clinical Center, Fudan University, Shanghai 201508, China \\ ${ }^{2}$ Department of Infectious Diseases, Huashan Hospital, Fudan University, Shanghai 200040, China \\ Correspondence to: Liang Chen, email: chenliang@shaphc.org \\ Qiang Li, email: liqiang66601@163.com
}

Keywords: chronic hepatitis B, non-alcoholic fatty liver disease, gamma-glutamyl transpeptidase-to-platelet ratio, liver fibrosis, non-invasive marker

Received: February 10, 2017

Accepted: March 04, 2017

Published: March 13, 2017

Copyright: Li et al. This is an open-access article distributed under the terms of the Creative Commons Attribution License (CC-BY), which permits unrestricted use, distribution, and reproduction in any medium, provided the original author and source are credited.

\section{ABSTRACT}

Background/Aim: The gamma-glutamyl transpeptidase-to-platelet ratio (GPR) is a novel serum model, which was reported more accurate than aspartate transaminaseto-platelet ratio index (APRI) and fibrosis index based on four factors (FIB-4) for diagnosing significant fibrosis and cirrhosis in HBV mono-infection in West Africa. We aimed to evaluate the diagnostic performance of GPR for liver fibrosis in patients with chronic hepatitis B (CHB) and non-alcoholic fatty liver disease (NAFLD).

Results: Of 131 patients, $41(31.3 \%), 20(15.3 \%)$, and $10(7.6 \%)$ were classified as having significant fibrosis, severe fibrosis and cirrhosis, respectively. To predict significant fibrosis, the AUROC of GPR was higher than that of APRI (0.86 vs 0.75 , $p=0.001)$ and FIB-4 $(0.86$ vs $0.66, p<0.001)$. To predict severe fibrosis, the AUROC of GPR was also higher than that of APRI (0.89 vs $0.77, p=0.002)$ and FIB-4 (0.89 vs $0.72, p=0.001$ ). To predict cirrhosis, no difference was found between the AUROC of GPR and that of APRI (0.92 vs $0.86, p=0.104)$.

Materials and Methods: 131 patients with CHB-NAFLD were included, and the diagnostic performances of GPR, APRI and FIB-4 were compared by receiver operating characteristic (ROC) curves and the area under ROC curves (AUROCs).

Conclusions: The GPR could be used as a non-invasive marker to predict liver fibrosis and cirrhosis in CHB-NAFLD individuals.

\section{INTRODUCTION}

Chronic hepatitis B (CHB), a disease caused by hepatitis B virus (HBV), is a leading cause for cirrhosis and hepatocellular carcinoma (HCC) [1]. Non-alcoholic fatty liver disease (NAFLD) covers a spectrum ranging from simple steatosis to steatohepatitis, and cirrhosis [2]. The prevalence of CHB is approximately $0.5 \%$ in the United States, $7 \%$ in China, and $10 \%$ in African countries [1]. The prevalence of NAFLD varies from $20 \%$ to $51 \%$, depending on the study population [3]. The increasing prevalence of NAFLD has resulted in the increased coexistence of CHB and NAFLD. In developed countries, NAFLD was observed in $20 \%$ of CHB patients $[4,5]$.

Lemoine et al propose a novel fibrosis model- the gamma-glutamyl transpeptidase (GGT) to platelet ratio
(GPR) - as a routinely available test that could identify patients with significant fibrosis or cirrhosis with higher diagnostic performance than aspartate transaminase (AST)to-platelet ratio index (APRI) and fibrosis index based on four factors (FIB-4) in HBV mono-infection patients in West Africa [6]. Schiavon and colleagues subsequently reported that the GPR showed an acceptable diagnostic performance for the detection of liver fibrosis in Brazilian patients with CHB, but it does not add any advantage over APRI and FIB-4 [7]. In the subsequent study, Lemoine confirmed that the GPR predicts significant fibrosis and cirrhosis with higher diagnostic performance than APRI and FIB-4, in a large cohort of $721 \mathrm{HBV}$ mono-infected Gambian patients using Fibroscan measures as a reference [8]. The heterogeneous populations may explain partly the discrepancies. Most of patients in the study by Schiavon 
et al are HBeAg seropositive (53\%) and high HBV DNA levels (median, $5.0 \log 10$ copies/ml) [7]; however, most of patients in the study by Lemoine et al. are $\mathrm{HBeAg}$ seronegative $(96 \%)$ and low HBV DNA levels (median, $2.6 \log 10$ copies $/ \mathrm{ml}$ ) [6]. The heterogeneous references for liver fibrosis may be another reason for the discrepancies. In the study by Schiavon et al, the evaluation of liver fibrosis was based on liver biopsy [7]; however, the 721 HBV mono-infected patients in the study by Lemoine et al used Fibroscan measures as a reference [8].

The above-mentioned studies excluded conditions that might predispose to altered GGT or platelet counts, including excessive alcohol consumption, accompanied by NAFLD, co-infection with HCV, HDV or HIV, and so on [6]. Consequently, high diagnostic accuracy of the GPR may not be applicative in patients with such conditions. Some researchers have done studies to validate the diagnostic accuracy of the GPR in CHB patients with such conditions. For example, Boyd et al reported that in a French HBV/HIV co-infected cohort, the GPR showed reasonable performance for identifying significant liver fibrosis [9]. Stockdale and colleagues subsequently reported that the GPR shows poor correlation with FibroScan measurements of liver fibrosis in HBV/HIV co-infected patients in West Africa [10].

At present, few studies have evaluated the performance of GPR for the diagnosis of liver fibrosis and cirrhosis in patients with CHB and NAFLD (CHBNAFLD). To fill this research gap, we evaluated the diagnostic performance of GPR for significant fibrosis, severe fibrosis, and cirrhosis in one hundred and thirtyone CHB-NAFLD patients, and compared with APRI and FIB-4 scores.

\section{RESULTS}

\section{Baseline characteristics of the patients}

The baseline characteristics of enrolled patients were shown in Table 1. The majority of enrolled patients were male $(72.5 \%)$, HBeAg positive $(66.4 \%)$, and middle-aged (39 \pm 10 years). The median HBV DNA, ALT, AST, and GGT were $5.6 \log 10$ copies/ml (IQR $=3.5-7.5), 47 \mathrm{IU} / \mathrm{L}(\mathrm{IQR}=29-70), 30 \mathrm{IU} / \mathrm{L}(\mathrm{IQR}=$ 24-37), and 45 IU/L (IQR $=16-57)$, respectively; and the mean platelet count was $182 \times 10^{9} / \mathrm{L}$. The Median GPR, APRI, and FIB-4 scores were 0.40 (IQR $=0.17-0.77)$, $0.44(\mathrm{IQR}=0.32-0.69)$, and $0.98(\mathrm{IQR}=0.68-1.43)$.

The METAVIR inflammation stage was distributed as follows: $\mathrm{A} 0=11(8.4 \%) ; \mathrm{A} 1=47(35.9 \%) ; \mathrm{A} 2=58$ $(44.3 \%)$; and A3 = 15 (11.5\%). The METAVIR fibrosis stage was distributed as follows: $\mathrm{F} 0=13(9.9 \%)$; $\mathrm{F} 1=77(58.8 \%) ; \mathrm{F} 2=21(16.0 \%) ; \mathrm{F} 3=10(7.6 \%) ;$ and $\mathrm{F} 4=10(7.6 \%)$. The hepatic steatosis stage was distributed as follows: $\mathrm{G} 1=65(49.6 \%) ; \mathrm{G} 2=41(31.3 \%) ; \mathrm{G} 3=15$ $(11.5 \%)$; and G4 = 10 (7.6\%). Of 131 enrolled patients,
$41(31.3 \%), 20(15.3 \%)$, and $10(7.6 \%)$ were classified as having significant fibrosis, severe fibrosis, and cirrhosis, respectively.

\section{Correlations between noninvasive markers and METAVIR fibrosis stages}

The correlations of noninvasive markers with METAVIR fibrosis stages were analysed using the Spearman test (Table 2). The GGT levels had a positive correlation with METAVIR fibrosis stages (correlation coefficient $\mathrm{r}=0.57, p<0.001$ ), platelet count was negatively correlated $(\mathrm{r}=-0.32, p<0.001)$. The METAVIR fibrosis stages were positive correlated with GPR $(\mathrm{r}=0.60, p<0.001)$, APRI $(\mathrm{r}=0.39, p<0.001)$, and FIB-4 ( $\mathrm{r}=0.25, p=0.004)$. The association between METAVIR fibrosis stages and noninvasive makers was presented in Figure 1.

\section{Correlations between noninvasive markers and liver steatosis levels}

The correlations of noninvasive markers with liver steatosis levels were presented in Table 3. The liver steatosis levels had no correlation with GGT $(\mathrm{r}=0.03$, $p=0.701)$, platelet count $(\mathrm{r}=-0.13, p=0.135), \mathrm{GPR}$ $(\mathrm{r}=0.08, p=0.389)$, APRI $(\mathrm{r}=0.13, p=0.142)$, and FIB-4 $(\mathrm{r}=0.15, p=0.098)$.

\section{Diagnostic performances of noninvasive models for liver fibrosis and cirrhosis}

The ROC curves of GPR, APRI, and FIB-4 for significant fibrosis (A), severe fibrosis (B), and cirrhosis (C), were shown in Figure 2. After estimating the performance to predict significant fibrosis, the AUROC of GPR was significantly higher than that of APRI $(0.86$ vs $0.75, p=0.001)$ and FIB-4 (0.86 vs $0.66, p<0.001)$ (Table 4). To predict severe fibrosis, the AUROC of GPR was also higher than that of APRI (0.89 vs 0.77 , $p=0.002)$ and FIB-4 (0.89 vs 0.72, $p=0.001)$ (Table 4). For the diagnosis of cirrhosis, the AUROC of GPR was significantly higher than FIB-4 (0.92 vs 0.73, $p<0.001)$. However, no statistical difference was found between GPR and APRI (0.92 vs 0.86, $p=0.104)$ in predicting cirrhosis (Table 4).

\section{Diagnostic thresholds of noninvasive models for liver fibrosis and cirrhosis}

Diagnostic thresholds of noninvasive models for liver fibrosis and cirrhosis were presented in Table 5. Maximizing Youden Index, the optimal cut-offs of GPR were $0.49,0.62$, and 0.74 , for the diagnosis of significant fibrosis (the corresponding sensitivity, specificity, PPV, and NPV was $83 \%, 80 \%, 65 \%$, and $91 \%$, respectively), severe 
Table 1: Baseline characteristics of the study population

\begin{tabular}{lc}
\hline & Total $(\boldsymbol{n}=\mathbf{1 3 1})$ \\
\hline Male $(n, \%)$ & $95(72.5 \%)$ \\
Age (year) & $39 \pm 10$ \\
HBeAg positive, $n(\%)$ & $87(66.4 \%)$ \\
HBV DNA (log10 copies/ml) & $5.6(3.5-7.5)$ \\
ALT (IU/L) & $47(29-70)$ \\
AST (IU/L) & $30(24-37)$ \\
GGT (IU/L) & $45(16-57)$ \\
Platelet count $(10 \% / \mathrm{L})$ & $182 \pm 61$ \\
GPR & $0.40(0.17-0.77)$ \\
APRI & $0.44(0.32-0.69)$ \\
FIB-4 & $0.98(0.68-1.43)$ \\
Median BMI (kg/m2) & $26(23-28)$ \\
METAVIR Inflammation stage (A0/A1/A2/A3) & $11(8.4 \%) / 47(35.9 \%) / 58(44.3 \%) / 15(11.5 \%)$ \\
METAVIR Fibrosis stage (F0/F1/F2/F3/F4) & $13(9.9 \%) / 77(58.8 \%) / 21(16.0 \%) / 10(7.6 \%) / 10(7.6 \%)$ \\
Hepatic steatosis stage (G1/G2/G3/G4) & $65(49.6 \%) / 41(31.3 \%) / 15(11.5 \%) / 10(7.6 \%)$ \\
\hline
\end{tabular}

$\mathrm{HBeAg}$, hepatitis B e antigen; ALT, alanine transaminase; AST, aspartate transaminase; GGT, gamma-glutamyl transpeptidase; GPR, GGT to platelet ratio index; APRI, AST to platelet ratio index; FIB-4, fibrosis index based on the 4 factors; BMI, body mass index.

Table 2: Correlations between noninvasive markers and liver fibrosis stages

\begin{tabular}{|c|c|c|}
\hline Variables & Spearman's $r$ & $P$ value \\
\hline GGT (IU/L) & 0.57 & $<0.001$ \\
\hline Platelet count $\left(10^{9} / \mathrm{L}\right)$ & -0.32 & $<0.001$ \\
\hline GPR & 0.60 & $<0.001$ \\
\hline APRI & 0.39 & $<0.001$ \\
\hline FIB-4 & 0.25 & 0.004 \\
\hline
\end{tabular}

GGT, gamma-glutamyl transpeptidase; GPR, GGT to platelet ratio index; APRI, aspartate transaminase to platelet ratio index; FIB-4, fibrosis index based on 4 factors; Spearman's r, correlation coefficient.

fibrosis (the corresponding sensitivity, specificity, PPV, and NPV was $90 \%, 75 \%, 39 \%$, and $98 \%$, respectively) and cirrhosis (the corresponding sensitivity, specificity, PPV, and NPV was $100 \%, 81 \%, 30 \%$, and $100 \%$, respectively), respectively. The optimal cut-offs of APRI were 0.41 , 0.44 , and 0.55 , for the diagnosis of significant fibrosis (the corresponding sensitivity, specificity, PPV, and NPV was $80 \%, 59 \%, 47 \%$, and $87 \%$, respectively), severe fibrosis (the corresponding sensitivity, specificity, PPV, and NPV was $80 \%, 56 \%, 25 \%$, and $94 \%$, respectively) and cirrhosis (the corresponding sensitivity, specificity, PPV, and NPV was $100 \%, 69 \%, 21 \%$, and $100 \%$, respectively), respectively.

\section{DISCUSSION}

HBV and NAFLD are highly co-endemic in China, and liver fibrosis is a common pathological process in CHB-NAFLD patients. Among CHB-NAFLD patients, those with significant fibrosis or cirrhosis are at increased risk for liver de-compensation, hepatocellular carcinoma (HCC), and death [1]. To reduce the disease burden, it is critical to identify patients with significant fibrosis or cirrhosis, and treat them timely. The early detection of significant fibrosis or cirrhosis is an essential step for CHB-NAFLD patients in deciding treatment commencement, course of treatment, and prognosis. Liver 
Table 3: Correlations between noninvasive markers and liver steatosis levels

\begin{tabular}{|c|c|c|}
\hline Variables & Spearman's $r$ & $P$ value \\
\hline GGT (IU/L) & 0.03 & 0.701 \\
\hline Platelet count $\left(10^{9} / \mathrm{L}\right)$ & -0.13 & 0.135 \\
\hline GPR & 0.08 & 0.389 \\
\hline APRI & 0.13 & 0.142 \\
\hline FIB-4 & 0.15 & 0.098 \\
\hline
\end{tabular}

GGT, gamma-glutamyl transpeptidase; GPR, GGT to platelet ratio index; APRI, aspartate transaminase to platelet ratio index; FIB-4, fibrosis index based on 4 factors; Spearman's r, correlation coefficient.

Table 4: Diagnostic performances of noninvasive models for liver fibrosis and cirrhosis

\begin{tabular}{|c|c|c|c|c|c|c|}
\hline & \multicolumn{2}{|c|}{ Significant fibrosis } & \multicolumn{2}{|c|}{ Severe fibrosis } & \multicolumn{2}{|c|}{ Cirrhosis } \\
\hline & AUROC & $(95 \%$ CI $)$ & AUROC & $(95 \%$ CI $)$ & AUROC & $(95 \% \mathrm{CI})$ \\
\hline GPR & 0.86 & $(0.79-0.91)$ & 0.89 & $(0.82-0.94)$ & 0.92 & $(0.87-0.96)$ \\
\hline APRI & 0.75 & $(0.66-0.82)$ & 0.77 & $(0.68-0.84)$ & 0.86 & $(0.79-0.92)$ \\
\hline FIB-4 & 0.66 & $(0.57-0.74)$ & 0.72 & $(0.70-0.80)$ & 0.73 & $(0.65-0.80)$ \\
\hline \multicolumn{7}{|l|}{$\begin{array}{l}\text { Comparison of } \\
\text { AUROC }\end{array}$} \\
\hline GPR and APRI & \multicolumn{2}{|c|}{$p=0.001$} & \multicolumn{2}{|c|}{$p=0.002$} & \multicolumn{2}{|c|}{$p=0.104$} \\
\hline GPR and FIB-4 & \multicolumn{2}{|c|}{$p<0.001$} & \multicolumn{2}{|c|}{$p=0.001$} & \multicolumn{2}{|c|}{$p<0.001$} \\
\hline APRI and FIB-4 & \multicolumn{2}{|c|}{$p=0.048$} & \multicolumn{2}{|c|}{$p=0.46$} & \multicolumn{2}{|c|}{$p=0.039$} \\
\hline
\end{tabular}

GPR, gamma-glutamyl transpeptidase to platelet ratio index; APRI, aspartate transaminase to platelet ratio index; FIB-4, fibrosis index based on the 4 factors; AUROC, the area under the receiver operating characteristic curve; CI, confidence interval.
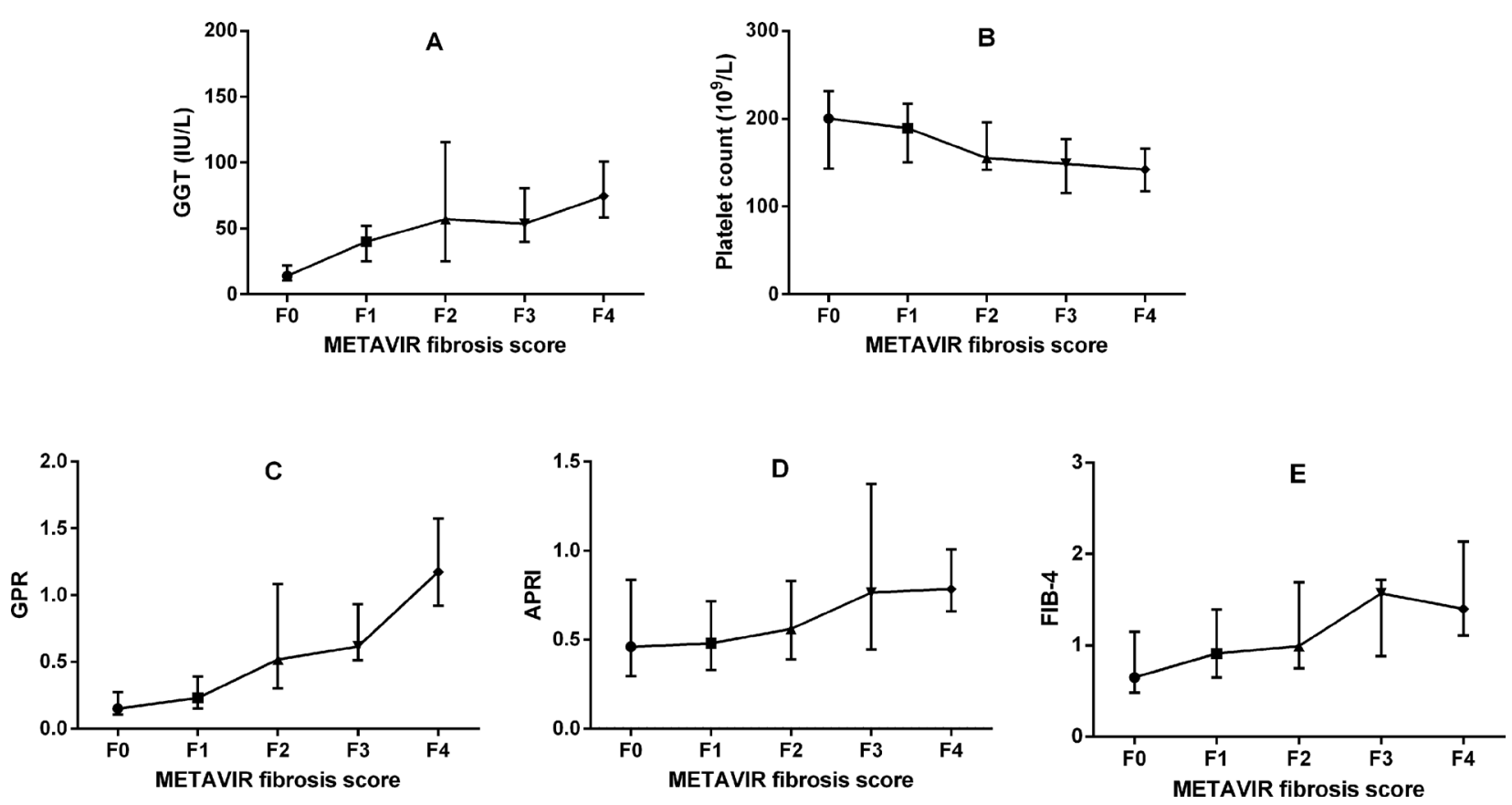

Figure 1: Association between METAVIR fibrosis stages and noninvasive markers. GGT, gamma-glutamyl transpeptidase; GPR, GGT to platelet ratio index; APRI, aspartate transaminase to platelet ratio index; FIB-4, fibrosis index based on the 4 factors. 
biopsy is the gold standard for assessment of liver fibrosis, but limited for its invasiveness, expensive procedure, and potentially complications.

In this study, we observed that the GPR had a higher performance compared to other commonly used models (APRI and FIB-4) for diagnosing significant fibrosis and severe fibrosis (all $p<0.05$ ) in CHB-NAFLD patients. For the diagnosis of cirrhosis, no statistical difference was found between GPR and APRI $(p=0.104)$. This study indicated that the GPR, which shows application prospect in HBV mono-infection or HBV/HIV co-infection patients in West Africa, also could be used to identify significant fibrosis, severe fibrosis, and cirrhosis in CHB-NAFLD patients. In this study, the liver steatosis levels had no correlation with GGT, platelet count, and GPR, along with no correlation between steatosis and fibrosis, which might help explain why the GPR works in CHB-NAFLD patients.

The GPR cut-offs in this study ( $\geq$ F2, 0.49; $\geq$ F3, $0.62 ;=F 4,0.74)$ were higher than those obtained in HBV mono-infection patients in West Africa $(\geq F 2,0.32 ; \geq F 3$, $0.32 ;=\mathrm{F} 4,0.56)$ [8]. As GGT levels were higher overall in CHB-NAFLD patients in this study, compared with HBV mono-infection patients in the study by Lemoine et al (45 vs $36 \mathrm{IU} / \mathrm{L}$ ) [8], and the need for modified thresholds would likely stem from elevated GGT levels. Kumar et al
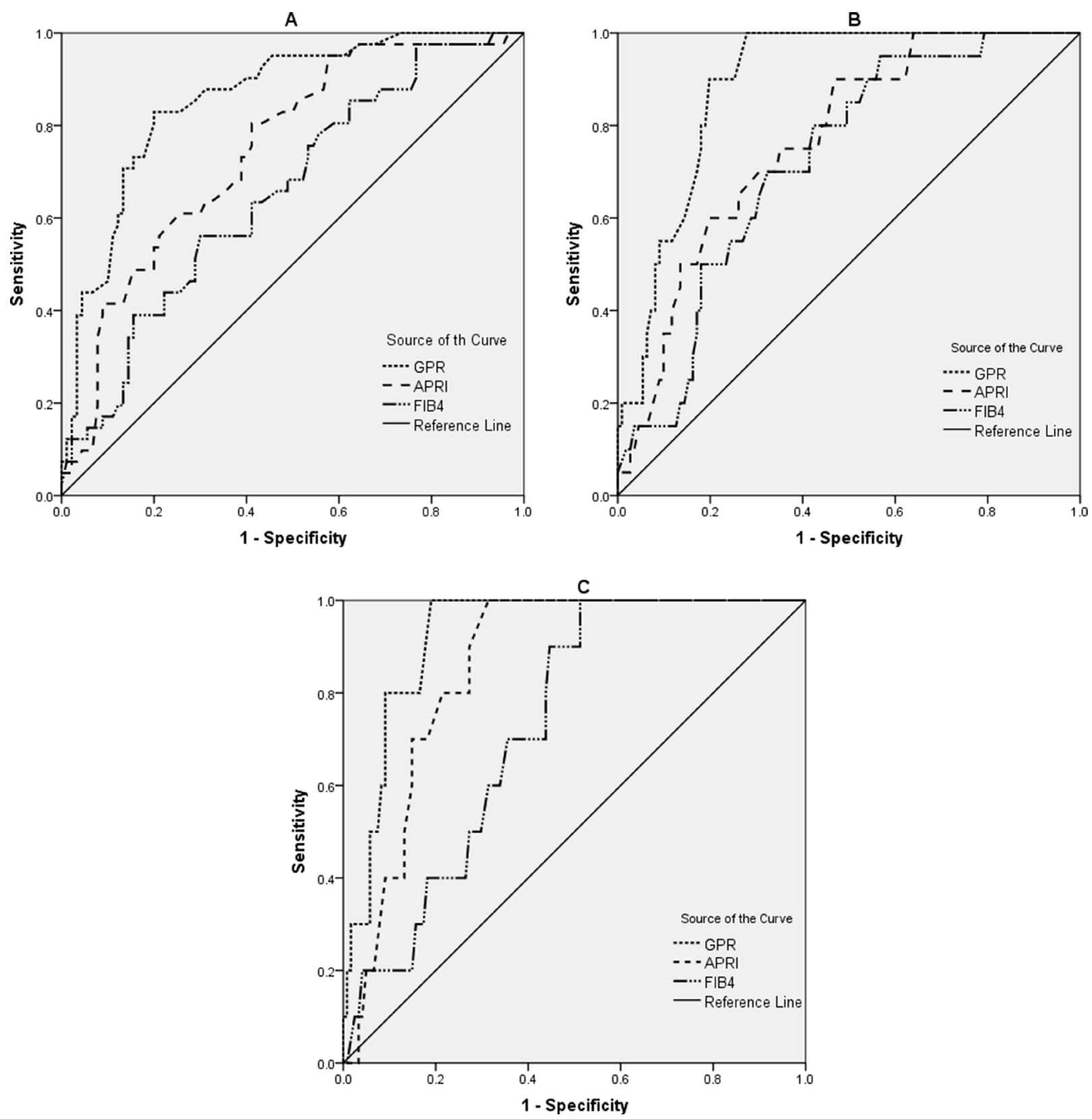

Figure 2: ROC curves for significant fibrosis (A), severe fibrosis $(\mathbf{B})$, and cirrhosis $(\mathbf{C})$. GPR, gamma-glutamyl transpeptidase to platelet ratio index; APRI, aspartate transaminase to platelet ratio index; FIB-4, fibrosis index based on the 4 factors. 
Table 5: Diagnostic thresholds of noninvasive models for liver fibrosis and cirrhosis

\begin{tabular}{lcccccccc}
\hline & Cut-offs & $\begin{array}{c}\text { Youden } \\
\text { Index }\end{array}$ & $\begin{array}{c}\text { Sensitivity } \\
(\%)\end{array}$ & $\begin{array}{c}\text { Specificity } \\
(\%)\end{array}$ & $\begin{array}{c}\text { PPV } \\
(\%)\end{array}$ & NPV (\%) & PLR & NLR \\
\hline GPR & & & & & & & & \\
$\geq$ F2 & 0.49 & 0.63 & 83 & 80 & 65 & 91 & 4.15 & 0.21 \\
$\geq$ F3 & 0.62 & 0.65 & 90 & 75 & 39 & 98 & 3.57 & 0.13 \\
= F4 & 0.74 & 0.81 & 100 & 81 & 30 & 100 & 5.26 & 0 \\
APRI & & & & & & & & \\
>F2 & 0.41 & 0.39 & 80 & 59 & 47 & 87 & 1.96 & 0.33 \\
$\geq$ F3 & 0.44 & 0.36 & 80 & 56 & 25 & 94 & 1.81 & 0.36 \\
= F4 & 0.55 & 0.69 & 100 & 69 & 21 & 100 & 3.18 & 0 \\
FIB-4 & & & & & & & & \\
$\geq$ F2 & 0.77 & 0.22 & 86 & 36 & 37 & 84 & 1.32 & 0.41 \\
$\geq$ F3 & 0.83 & 0.38 & 95 & 43 & 23 & 98 & 1.67 & 0.12 \\
= F4 & 0.91 & 0.49 & 100 & 49 & 14 & 100 & 1.95 & 0 \\
\hline
\end{tabular}

GPR, gamma-glutamyl transpeptidase to platelet ratio index; APRI, aspartate transaminase to platelet ratio index; FIB-4, fibrosis index based on the 4 factors; PPV, positive predictive value; NPV, negative predictive value; PLR, positive likelihood ratio; NLR, negative likelihood ratio. The GPR cut-offs obtained in HBV mono-infection patients in West Africa by Lemoine et al. were 0.32 for $\geq F 2,0.32$ for $\geq F 3$, and 0.56 for $=F 4$, respectively.

found that the CHB-NAFLD patients tend to present with higher transaminase and GGT levels compared with HBV mono-infection [11]. In addition, obesity could increase GGT; and CHB-NAFLD patients in this study have higher BMI (26 vs $22 \mathrm{~kg} / \mathrm{m} 2)$ than HBV mono-infection patients in the study by Lemoine et al [8]. Secondly, the prevalence of fibrosis $(31.3 \%$ for $\geq \mathrm{F} 2 ; 15.3 \%$ for $\geq \mathrm{F} 3 ; 7.6 \%$ for $=$ F4) in this study was lower than what was obtained by Lemoine et al $(39 \%$ for $\geq \mathrm{F} 2 ; 32 \%$ for $\geq \mathrm{F} 3 ; 15 \%$ for $=\mathrm{F} 4)$ [8]. Difference between the GPR cut-offs may be related to differences in prevalence of liver fibrosis in the studied populations, known as the spectrum bias $[12,13]$.

GPR had good NPVs for excluding significant fibrosis (91\%), severe fibrosis (98\%), and cirrhosis (100\%), respectively; but low PPVs for diagnosing significant fibrosis $(65 \%)$, severe fibrosis $(39 \%)$, and cirrhosis (30\%), respectively. Likewise, in this study, APRI and FIB-4 also had low PPVs for diagnosing significant fibrosis ( $47 \%$ and $37 \%$, respectively), severe fibrosis $(25 \%$ and $23 \%$, respectively), and cirrhosis $(21 \%$ and $14 \%$, respectively), respectively. In fact, the low PPVs were common problem with noninvasive fibrosis models. According to the recent WHO HBV guideline, the PPV was low (less than 50\%) for all non-invasive tests for the diagnosis of liver fibrosis and cirrhosis, and FibroScan had a relatively higher PPV (42\%) than APRI using either a high or low cut-off $(26 \%$ and 22\%) [14]. Although the PPVs of all noninvasive fibrosis models were low, GPR had a relatively higher PPVs compared with APRI and FIB-4, for the diagnosis of significant fibrosis $(65 \%, 47 \%$, and $37 \%$, respectively), severe fibrosis $(39 \%, 25 \%$, and $23 \%$, respectively), and cirrhosis $(30 \%, 21 \%$, and $14 \%$, respectively), respectively.
It is important to note that the APRI threshold for the diagnosis of cirrhosis $(>2.0)$ recommended by the WHO HBV guideline was unsuitable in CHB-NAFLD population, such that none of the ten patients with cirrhosis were correctly identified. This implies that $100 \%$ of patients who had cirrhosis would be erroneously categorized as patients without cirrhosis by APRI $>2$. In this study, the optimal cut-off of APRI is 0.55 to diagnose cirrhosis, and the corresponding sensitivity, specificity, PPV, and NPV were $100 \%, 69 \%, 21 \%$, and $100 \%$, respectively. Obviously, APRI $>0.55$ is more appropriate for screening cirrhosis and selection of candidates for liver biopsy in CHB-NAFLD population. Compared with HBV monoinfection patients, the different magnitude of inflammation and related ALT levels observed in CHB-NAFLD patients, that might render the different APRI cut-offs. Two recent studies, showing different pathogenesis and different patterns of fibrosis according to different causes of chronic liver diseases, also justify the need for different cut-offs of systems for assessment of fibrosis from different causes $[15,16]$.

It is undeniable that this study has some limitations. First, there were few patients with F3 and F4 fibrosis $(n=20)$ and perhaps there was lack of power to determine a statistical difference between APRI and GPR AUROCs at the F4 level. Second, our study population, with higher prevalence of $\mathrm{HBeAg}$-positivity and higher proportion of male, might not be fully representative of CHB-NAFLD patients. Third, we do not compare the performance of FibroScan to GPR because of the FibroScan measurements have not been promoted widely in China. Fourth, we do not compare the performance of FibroTest, Hepascore and FibroMeter to GPR because these models were protected by patents, and some laboratory tests which are necessary 
for the calculations of these models were not available in our hospital. Fifth, there is no external validation, and the predictive capacity of the GPR thresholds obtained in this study need to be further validated in other cohorts of similar study population.

Notwithstanding these limitations, we validate that the GPR could be used as a non-invasive marker to predict liver fibrosis and cirrhosis for CHB-NAFLD individuals, especially when compared with APRI and FIB-4. Certainly, we hope that further evaluations will be conducted in CHB-NAFLD patients more broadly.

\section{MATERIALS AND METHODS}

\section{Study design and patients}

Three hundred and fourteen consecutive patients with CHB and biopsy-proven fatty liver disease (defined as the presence of more than 5\% steatosis of hepatocytes) who underwent liver biopsies and routine laboratory tests at Shanghai Public Health Clinical Center, Shanghai, China between May 2008 and January 2017 were retrospectively screened. $\mathrm{CHB}$ was defined as the persistent presence of hepatitis B surface antigen (HBsAg) for more than 6 months [17]. The fatty liver disease was considered to be non-alcoholic in origin if the clinical records indicated that the patient was felt to either be totally abstinent or to consume less than approximately $20 \mathrm{~g}$ of alcohol daily [18]. Patients with the following conditions were excluded: (1) alcohol consumption
$>20 \mathrm{~g} /$ day $(n=121)$; (2) antiviral therapy history $(n=26)$; (3) co-infection with hepatitis C virus (HCV), hepatitis D virus (HDV), or human immunodeficiency virus (HIV) $(n=33)$; (4) accompanied by autoimmune liver disease $(n=3)$. Finally, 131 treatment-naïve patients with CHBNAFLD were included. Figure 3 summarized the flow diagram of the study population.

All patients signed the informed consent before liver biopsy, and all clinical procedures were in accordance with the Helsinki declaration of 1975 , as revised in 1983. The study protocol was permitted by the ethics committee of Shanghai Public Health Clinical Center.

\section{Liver histological score}

Liver biopsy was performed using ultrasound localization. Liver samples were formalin-fixed and paraffin-embedded. A minimum of $15 \mathrm{~mm}$ of liver tissue with at least 6 portal tracts is considered sufficient for liver histological scoring. Liver histology was interpreted by two liver pathologists. In case of discrepancies, slides were reviewed by a third highly experienced hepatopathologist.

The METAVIR scoring system was adopted as the histological standard of liver fibrosis, which was classified into five stages: F0, no fibrosis; F1, portal fibrosis without septa; F2, portal fibrosis with rare septa; F3, numerous septa without cirrhosis; and F4, cirrhosis [19]. Steatosis was defined as the presence of more than $5 \%$ steatosis of hepatocytes, and the degree of steatosis was semiquantitatively graded from 0 to 4 as follows: grade 0: less

\section{4 consecutive patients with $\mathrm{CHB}$ and biopsy-proven fatty liver disease between May 2008 and January 2017}

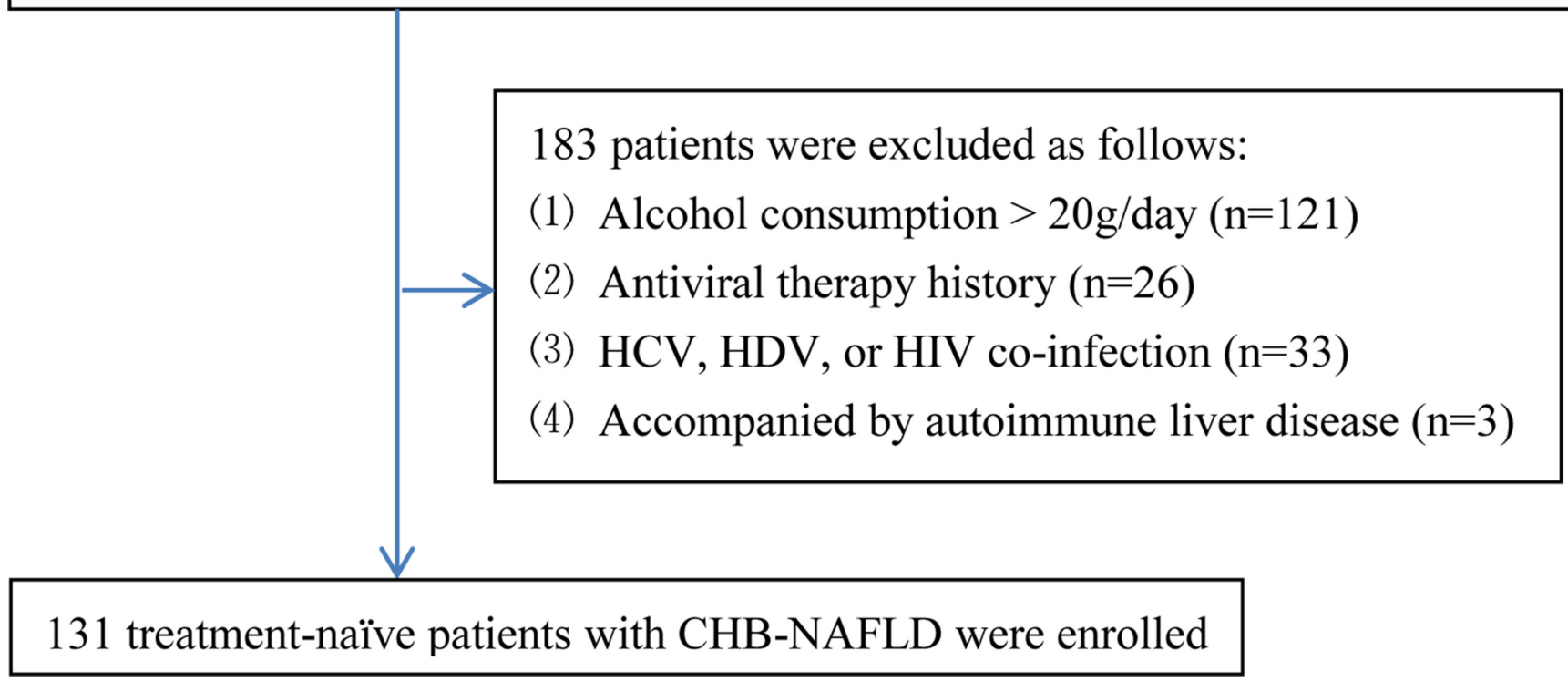

Figure 3: Flow diagram of the study population. CHB, chronic hepatitis B; NAFLD, non-alcoholic fatty liver disease; HCV, hepatitis C virus; HDV, hepatitis D virus; HIV, human immunodeficiency virus. 
than $5 \%$ steatosis; grade $1: 6 \%$ to $25 \%$, grade $2: 26 \%$ to $50 \%$, grade 3: $51 \%$ to $75 \%$, and grade 4 : more than $76 \%$ [20]. Significant fibrosis was defined as fibrosis stage $\geq F 2$, severe fibrosis was defined as fibrosis stage $\geq$ F3, and cirrhosis was defined as fibrosis stage $=$ F4. Steatohepatitis was defined by the minimal criteria of hepatic steatosis and scattered, mainly lobular inflammation with or without Mallory bodies, cytologic ballooning, and perisinusoidal fibrosis [21].

\section{Routine laboratory tests}

Fasting blood samples were obtained, and routine laboratory tests were performed the day before liver biopsy. The serological markers of HBV were detected with enzyme-linked immune-sorbent assay kits (ARCHITECT i2000 SR; Abbott, Wiesbaden, Germany). The serum biochemical parameters including alanine transaminase (ALT), AST, and GGT were measured by full automated biochemistry analyzer (7600 Series; Hitachi, Tokyo, Japan). Platelet count was detected with automated hematology analyzer (XT-2000i, Sysmex, Kobe, Japan). HBV DNA was quantified by real-time PCR (ABI 7500; Applied Biosystems, Foster City, USA) with the lowest detection limit at 500 copies $/ \mathrm{ml}$.

\section{Noninvasive models calculation}

The formulas for GPR, APRI, and FIB-4 are as follows: (1) $\mathrm{GPR}=(\mathrm{GGT}(\mathrm{IU} / \mathrm{L}) / \mathrm{ULN}$ of GGT)/platelet $\operatorname{count}\left(10^{9} / \mathrm{L}\right) \times 100 ;(2)$ APRI $=($ AST $(\mathrm{IU} / \mathrm{L}) / \mathrm{ULN}$ of AST $) /$ platelet count $\left(10^{9} / \mathrm{L}\right) \times 100$; $(3)$ FIB-4 $=($ age $($ years $) \times$ AST $(\mathrm{IU} / \mathrm{L})) /\left(\right.$ platelet count $\left.\left(10^{9} / \mathrm{L}\right) \times(\text { ALT }(\mathrm{IU} / \mathrm{L}))^{1 / 2}\right)$.

Note: ULN of AST $=40 \mathrm{IU} / \mathrm{L}$; ULN of GGT $=50$ IU/L.

\section{Statistical analysis}

Normality tests of baseline data were performed by Kolmogorov-Smirnov test. The baseline data of enrolled patients was presented as follows: normal distribution data as mean \pm standard deviation, non-normal distribution continuous data as median (interquartile range (IQR)), and categorical variables as number (percentage). The diagnostic performances of GPR, APRI, and FIB-4 were evaluated and compared by the receiver operating characteristic (ROC) curves and the area under the ROC curves (AUROCs) [22]. The ROC curve analysis and $Z$-test was, respectively, used to compute and compare AUROC. The optimal cut-offs were obtained by maximizing Youden index (sensitivity + specificity-1). Diagnostic accuracy was evaluated by sensitivity, specificity, positive predictive value (PPV), negative predictive value (NPV), positive likelihood ratio (PLR), and negative likelihood ratio (NLR). All significance tests were two tailed, and $p<0.05$ was considered statistically significant. All statistical analyses were carried out using the SPSS statistical software version 15.0 (SPSS Inc. Chicago, IL, USA) and MedCalc Statistical Software version 16.1 (MedCalc Software bvba, Ostend, Belgium).

\section{Authors' contributions}

Study concept and design: Qiang Li. Analysis and interpretation of data: Liang Chen, Chuan Lu, Weixia Li, Yuxian Huang and Qiang Li. Drafting of the manuscript: Qiang Li. Critical revision of the manuscript for important intellectual content: Liang Chen.

\section{ACKNOWLEDGMENTS}

This study was supported by grant No.SHDC12015129 from the ShenKang development center of Shanghai, and grant NO. 13401902100 from the science and technology commission of Shanghai.

\section{CONFLICTS OF INTEREST}

The authors declare no competing financial interests. The funding organizations are public institutions and had no role in the design and conduct of the study; collection, management, and analysis of the data; or preparation, review, and approval of the manuscript.

\section{FINANCIAL SUPPORT}

This study was supported by grant No.SHDC12015129 from the ShenKang development center of Shanghai, and grant NO. 13401902100 from the science and technology commission of Shanghai.

\section{REFERENCES}

1. Wu JF, Chang MH. Natural history of chronic hepatitis B virus infection from infancy to adult life-the mechanism of inflammation triggering and long-term impacts. J Biomed Sci. 2015; 22:92.

2. Anstee QM, Targher G, Day CP. Progression of NAFLD to diabetes mellitus, cardiovascular disease or cirrhosis. Nat Rev Gastroenterol Hepatol. 2013; 10:330-344.

3. Marengo A, Jouness RI, Bugianesi E. Progression and Natural History of Nonalcoholic Fatty Liver Disease in Adults. Clin Liver Dis. 2016; 20:313-324.

4. Bondini S, Kallman J, Wheeler A, Prakash S, Gramlich T, Jondle DM, Younossi ZM. Impact of non-alcoholic fatty liver disease on chronic hepatitis B. Liver Int. 2007; 27:607-611.

5. Thomopoulos KC, Arvaniti V, Tsamantas AC, Dimitropoulou D, Gogos CA, Siagris D, Theocharis GJ, Labropoulou-Karatza C. Prevalence of liver steatosis in patients with chronic hepatitis B: a study of associated factors and of relationship with fibrosis. Eur J Gastroenterol Hepatol. 2006; 18:233-237. 
6. Lemoine M, Shimakawa Y, Nayagam S, Khalil M, Suso P, Lloyd J, Goldin R, Njai HF, Ndow G, Taal M, Cooke G, D'Alessandro U, Vray $\mathrm{M}$, et al. The gamma-glutamyl transpeptidase to platelet ratio (GPR) predicts significant liver fibrosis and cirrhosis in patients with chronic HBV infection in West Africa. Gut. 2016; 65:1369-1376.

7. Schiavon LL, Narciso-Schiavon JL, Ferraz ML, Silva AE, Carvalho-Filho RJ. The gamma-glutamyl transpeptidase to platelet ratio (GPR) in HBV patients: just adding up? Gut. 2016.

8. Lemoine M, Thursz M, Mallet V, Shimakawa Y. Diagnostic accuracy of the gamma-glutamyl transpeptidase to platelet ratio (GPR) using transient elastography as a reference. Gut. 2017; 66:195-196.

9. Boyd A, Bottero J, Lacombe K. The gamma-glutamyl transpeptidase-to-platelet ratio as a predictor of liver fibrosis in patients co-infected with HBV and HIV. Gut. 2016; 65:718-720.

10. Stockdale AJ, Phillips RO, Geretti AM. The gamma-glutamyl transpeptidase to platelet ratio (GPR) shows poor correlation with transient elastography measurements of liver fibrosis in HIV-positive patients with chronic hepatitis B in West Africa. Response to: 'The gamma-glutamyl transpeptidase to platelet ratio (GPR) predicts significant liver fibrosis and cirrhosis in patients with chronic HBV infection in West Africa' by Lemoine et al. Gut. 2016; 65:882-884.

11. Kumar R, Boon-Bee GG. Chronic hepatitis B and fatty liver: Issues in clinical management. Clin Res Hepatol Gastroenterol. 2016; 40:755-759.

12. Ransohoff DF, Feinstein AR. Problems of spectrum and bias in evaluating the efficacy of diagnostic tests. N Engl J Med. 1978; 299:926-930.

13. Poynard T, Halfon P, Castera L, Munteanu M, ImbertBismut F, Ratziu V, Benhamou Y, Bourliere M, de Ledinghen V. Standardization of ROC curve areas for diagnostic evaluation of liver fibrosis markers based on prevalences of fibrosis stages. Clin Chem. 2007; 53:1615-1622.
14. World Health Organization. Guidelines for the Prevention, Care and Treatment of Persons with Chronic Hepatitis B Infection. 2015

15. Mason WS, Gill US, Litwin S, Zhou Y, Peri S, Pop O, Hong ML, Naik S, Quaglia A, Bertoletti A, Kennedy PT. HBV DNA Integration and Clonal Hepatocyte Expansion in Chronic Hepatitis B Patients Considered Immune Tolerant. Gastroenterology. 2016; 151:986-998.

16. Quaglia A, Alves VA, Balabaud C, Bhathal PS, BioulacSage P, Crawford JM, Dhillon AP, Ferrell L, Guido M, Hytiroglou P, Nakanuma Y, Paradis V, Snover DC, et al. Role of aetiology in the progression, regression, and parenchymal remodelling of liver disease: implications for liver biopsy interpretation. Histopathology. 2016; 68:953-967.

17. Terrault NA, Bzowej NH, Chang KM, Hwang JP, Jonas MM, Murad MH. AASLD guidelines for treatment of chronic hepatitis B. Hepatology. 2016; 63:261-283.

18. Mofrad P, Contos MJ, Haque M, Sargeant C, Fisher RA, Luketic VA, Sterling RK, Shiffman ML, Stravitz RT, Sanyal AJ. Clinical and histologic spectrum of nonalcoholic fatty liver disease associated with normal ALT values. Hepatology. 2003; 37:1286-1292.

19. Bedossa P, Poynard T. An algorithm for the grading of activity in chronic hepatitis $\mathrm{C}$. The METAVIR Cooperative Study Group. Hepatology. 1996; 24:289-293.

20. Matteoni CA, Younossi ZM, Gramlich T, Boparai N, Liu YC, McCullough AJ. Nonalcoholic fatty liver disease: a spectrum of clinical and pathological severity. Gastroenterology. 1999; 116:1413-1419.

21. Brunt EM, Janney CG, Di Bisceglie AM, NeuschwanderTetri BA, Bacon BR. Nonalcoholic steatohepatitis: a proposal for grading and staging the histological lesions. Am J Gastroenterol. 1999; 94:2467-2474.

22. Albeck MJ, Borgesen SE. [ROC-curve analysis. A statistical method for the evaluation of diagnostic tests]. Ugeskr Laeger. 1990; 152:1650-1653. 